\title{
Effect of carriers on the optical properties of AlGaAs/GaAs interdiffused quantum well lasers
}

\author{
E. Herbert Li \\ Department of Electrical and Electronic Engineering, University of Hong Kong \\ Pokfulam Road, Hong Kong.
}

\begin{abstract}
The carrier-induced effects in the change of refractive index on the $\mathrm{GaAs} / \mathrm{Al}_{\mathrm{w}} \mathrm{Ga}_{1-\mathrm{w}}$ as square quantum well (SqQW) and diffused quantum well (DFQW) was investigated. Band-filling, bandgap shrinkage, and free-carrier absorption were included. Carrier concentrations from $10^{16}$ to $10^{18} \mathrm{~cm}^{-3}$ were considered. The energy levels and their associated wavefunctions in the SqQW or DFQW structures are calculated by solving both the Schrödinger and the Poisson equations self-consistently. It is followed by the absorption change, which is defined as the difference between the absorption coefficient with carrier injection in QW and that without carrier injection. The refractive index change can be obtained by applying Kramers-Kronig Transformation. These results obtained are useful in the design of devices, such as lasers, optical phase, modulators and switches. Thus, it is important to know the carrier-induced energy shift in $\mathrm{GaAs} / \mathrm{Al}_{\mathrm{W}} \mathrm{Ga}_{1-\mathrm{w}}$ as quantum well structures.
\end{abstract}

Keywords: $\mathrm{AlGaAs} / \mathrm{GaAs}$, interdiffused quantum well, lasers, carrier effects, refractive index, absorption

\section{INTRODUCTION}

The quantum confinement of carriers in semiconductor quantum wells (QWs) and superlattices, which leads to the quantization of energy levels, has been studied extensively. These effects include linear and nonlinear transitions, with and without the application of a uniform electric or magnetic field, which will alter the position of the quantized energy levels and therefore contribute to the absorption coefficient and refractive index of the structure. However, most of the developments in QW devices were based on square (rectangular) well structures. It is expected that the ladder of the quantized energy levels are more sensitive to nonsquare quantum well shapes than to square well shapes. The properties of these nonsquare QWs are clearly of interest as promising candidates for their tailoring potential, and may give rise to interesting device properties ${ }^{1}$.

The potential profile of a QW produced by selective impurity induced compositional disordering can be changed from that of a square well to a nonsquare well so that the band gap, and therefore the 
optical and electronic properties of the structure, are modified. The disordering process increases the effective band gap and decreases the refractive index of the QW so that the implanted regions may be used to confine photons to the as-grown (ordered) part of the structure, i.e., this process may be used to form an optical waveguide ${ }^{2}$.

In fact, the effect of carriers in quantum wells has drawn a lot of attention. The interactions between carriers (electron-electron, hole-hole, and electron-hole) may cause the emission wavelength (energy) to shift, which is known as bandgap renormalization. This shift is important in the device designs anf applications. The confinement energy levels in the QW structure can be tailored by changing the well width as well as its shape with different compositions. These factors affect the shift of emission energy induced by injected carriers. So it is an interesting and important area to investigate in terms of the carrier induced energy shift and optical parameter change in $\mathrm{GaAs} / \mathrm{Al}_{\mathrm{W}} \mathrm{Ga}_{1-\mathrm{w}} \mathrm{As}$ quantum well structure.

In this project, the main aim is to investigate the effects of carrier-induced change in absorption coefficient as well as refractive index on the $\mathrm{GaAs} / \mathrm{Al}_{\mathrm{W}} \mathrm{Ga}_{1-\mathrm{w}} \mathrm{As} \mathrm{SqQW}$ and DFQW. In fact, little work has been reported in the corresponding research. However, this research has a considerable significance on the device design such as modulation doped field effect transistors, photodetectors, QW lasers, photonic switch, and modulators, etc..

\section{THEORY AND MODELLING}

At the end of the paper, we would estimate the carrier-induced changes in refractive index for $\mathrm{GaAs} / \mathrm{Al}_{\mathrm{W}} \mathrm{Ga}_{1-\mathrm{W}} \mathrm{As} \mathrm{QW}$. We will consider the case of electrical injection for carrier concentrations ranging from $10^{16}$ to $10^{18} / \mathrm{cm}^{3}$. Three carrier effects are included, namely : bandgap shrinkage, bandfilling, free-carrier absorption, where the mechanisms of these factors were extensively discussed in Ref.3. Here we consider one of these effects: Bandgap shrinkage.

In this case, both electrons and holes are treated self-consistently ${ }^{4}$. The $z$ direction, perpendicular to the quantum well plane, was chosen. The initial potential profile $V_{0, r}(z)$ of the DFQW for electrons, heavy and light holes are already given by Ref. 5

$$
-\frac{\hbar^{2}}{2} \frac{\mathrm{d}}{\mathrm{dz}}\left[\frac{1}{\mathrm{~m}^{*}{ }_{\perp \mathrm{r}}(\mathrm{z})} \frac{\mathrm{d} \mathrm{Y}_{\mathrm{rl}}(\mathrm{z})}{\mathrm{dz}}\right]+\mathrm{U}_{\mathrm{r}}(\mathrm{z}) \mathrm{Y}_{\mathrm{rl}}(\mathrm{z})=\mathrm{E}_{\mathrm{rl}} \mathrm{Y}_{\mathrm{rl}}(\mathrm{z})
$$

i.e. $U_{r}(z)=V_{o, r}(z)$. By solving equation (1) we can solve $E_{r l}$ and $\Psi_{r l}$, which are the energy level and wave function for the lth sublevel respectively. The electron, heavy and light hole distribution $\mathrm{n}_{\mathrm{e}}(\mathrm{z})$, $\mathrm{n}_{\mathrm{hh}}(\mathrm{z}), \mathrm{n}_{\mathrm{lh}}(\mathrm{z})$ respectively are given by Ref. 6

$$
\mathrm{n}_{\mathrm{i}}(\mathrm{z})=\sum_{\mathrm{M}} \frac{\mathrm{m}_{/ /}{ }^{*} \mathrm{k}_{\mathrm{B}} \mathrm{T}}{\mathrm{p} \hbar^{2} \mathrm{~L}_{\mathrm{z}}} \ln \left[1+\exp \left(\frac{\mathrm{E}_{\mathrm{Fi}}-\mathrm{E}_{\mathrm{r} \ell}}{\mathrm{k}_{\mathrm{B}} \mathrm{T}}\right)\right] \cdot\left|\mathrm{Y}_{\mathrm{r} \ell}\right|^{2}
$$


where $\mathrm{m} / /^{*}$ is parallel effective mass. $\mathrm{k}_{\mathrm{B}}$ is Boltzmann's constant, $\mathrm{T}$ is absolute room temperature, i.e. $\mathrm{T}$ $=300 \mathrm{~K}, \mathrm{~L}_{\mathrm{Z}}$ is the effective width in the $\mathrm{z}$ direction, $\mathrm{E}_{\mathrm{Fi}}$ is Fermi energy which has already been given in Ref. 3.

Once carrier spatial distributions $\mathrm{n}_{\mathrm{e}}, \mathrm{n}_{\mathrm{hh}}$ and $\mathrm{n}_{\mathrm{lh}}$ are obtained, we can then estimate many-body carrier effects, namely the Hartree term and exchange-correlation term. Hartree terms $\mathrm{V}_{\mathrm{He}}, \mathrm{V}_{\mathrm{Hhh}}$ and $\mathrm{V}_{\mathrm{Hlh}}$ are obtained from the Poisson equation, as in the following:

$$
\begin{aligned}
& \frac{\mathrm{d}^{2}}{\mathrm{dz}^{2}} \mathrm{~V}_{\mathrm{He}}(\mathrm{z})=-\frac{4 \pi \mathrm{e}}{\varepsilon}\left(\mathrm{n}_{\mathrm{hh}}(\mathrm{z})+\mathrm{n}_{\mathrm{lh}}(\mathrm{z})-\mathrm{n}_{\mathrm{e}}(\mathrm{z})\right) \\
& \mathrm{V}_{\mathrm{Hhh}}(\mathrm{z})=\mathrm{V}_{\mathrm{Hlh}}(\mathrm{z})=-\mathrm{V}_{\mathrm{He}}(\mathrm{z})
\end{aligned}
$$

$\varepsilon$ is the dielectric constant. The boundary condition is taken as $V_{H h h}(z), V_{H l h}(z)$ and $V_{H e}(z)$ equal zero at $z_{b}= \pm 50 \mathrm{~nm}$. With this condition, the Poisson equation can be solved without any difficulty. The exchange-correlation term $\mathrm{V}_{\mathrm{Xc}}$ is given in Ref. 7 by

$$
\mathrm{V}_{\mathrm{xc}, \mathrm{r}}(\mathrm{z})=-\frac{2}{\pi \alpha \mathrm{r}_{\mathrm{s}}}\left[1+0.7734 \mathrm{x} \ln \left(1+\frac{1}{\mathrm{x}}\right)\right] \mathrm{R}_{\mathrm{y}}
$$

The subscript $r$ stands for electron, heavy hole and light hole, $R_{y}$ is the effective Rydberg. The effective potential for carriers is given by the sum of the initial potential of the QW, Hartree term, and exchange-correlation term:

$$
\mathrm{U}_{\mathrm{r}}=\mathrm{V}_{\mathrm{o}, \mathrm{r}}+\mathrm{V}_{\mathrm{Hr}}+\mathrm{V}_{\mathrm{xc}, \mathrm{r}}
$$

Inserting new potential (5) into the Schrodinger equation (1), new energy levels and carrier distributions are obtained. By continuing this calculation loop until $\mathrm{E}_{\mathrm{rl}}$ values converge we obtain selfconsistent energy levels Ref. 4.

The absorption coefficient for the case of no carrier injection, $\alpha_{0}(\omega)$, will first be considered. Afterwards, the absorption coefficient with carrier injection, $\alpha(\omega)$, will then be determined, in which another carrier effect is included: Bandfilling.

Once the electron and hole envelope wavefunctions and subband energy levels of the QW structure have been determined, the linear absorption coefficient $\alpha(\omega)$ may be determined in terms of the imaginary part of the dielectric function $\varepsilon_{2}(\omega)$ using the relation:

$$
\alpha(\omega)=\frac{\omega \varepsilon_{2}(\omega)}{c_{0} n_{R}(\omega)}
$$


where $n_{R}(\omega)$ and $c_{O}$ are the refractive index of the inter-diffused $Q W$ and the speed of light, respectively. The incoming electromagnetic radiation is taken to be propagating parallel to the $\mathrm{x}-\mathrm{y}$ plane (perpendicular to $\mathrm{z}$ axis) of the QW layer, therefore $\alpha$ and $\varepsilon_{2}$ are anisotropic with polarization (TE or TM). Since we are interested in the QW quantization effect which manifests itself over a limited energy range above the bandgap, we use an $\varepsilon_{2}(\omega)$ calculation, which is based on the direct interband transitions around the absorption edge in the $\Gamma$-valley with a parabolic band, and three additional factors to take account of (i) the polarization, (ii) the 2D enhancement Sommerfeld factor, and (iii) the factor that includes the probability of the valance band state being occupied multiplied by the probability of the conduction band state being unoccupied. ${ }^{8}$ Thus the absorption coefficient $\alpha(\omega)$ becomes

$$
\alpha(\omega)=\frac{e^{2} m_{l /}^{*} w}{6 e_{o} c_{o} n_{r} m_{e} E_{C V}^{2} L_{z}} \tilde{M}_{o} \sum_{p \bullet q}\left|\left\langle Y_{C p} \mid Y_{V q}\right\rangle\right|^{2} I_{p q}(\hbar w)
$$

For the case of bandfilling, we include two terms $f_{C}$ and $f_{V}$ which are given by the Ferni-Dirac distribution functions ${ }^{3}$. Thus the absorption coefficient $\alpha(\omega)$ due to the induced carrier effect is obtained in the above manner.

After we calculate the absorption coefficient $\alpha_{O}(\omega)$ of the $\mathrm{GaAs}_{\mathrm{Al}} \mathrm{W}_{\mathrm{W}} \mathrm{Ga} 1-\mathrm{W} A \mathrm{As}$ QW without carrier injection, we are able to compute the change in absorption coefficient $\Delta \alpha$, which is given by:

$$
\Delta \alpha=\alpha(\omega)-\alpha_{0}(\omega)
$$

The change in refractive index $\Delta \mathrm{n}$ due to the change in absorption coefficient $\Delta \alpha$ can be calculated by the Kramers-Kronig Transformation, which is given in Ref. 9 by:

$$
\Delta \mathrm{n}(\mathrm{E})=6.28 \times 10^{-6} \mathrm{P} \int_{0}^{\infty} \frac{\Delta \alpha\left(\mathrm{E}^{\prime}\right)}{\left(\mathrm{E}^{\prime}\right)^{2}-\mathrm{E}^{2}} \mathrm{dE} \mathrm{E}^{\prime}
$$

where $\mathrm{P}$ stands for the principal Cauchy integral and $\mathrm{E}=\mathrm{h} \omega$ is the photon energy, the units of $\alpha$ and $E$ are $\mathrm{cm}^{-1}$ and $\mathrm{eV}$, respectively.

We now consider the last carrier effect: Free-carrier absorption [3]. Then, Eq. (9) becomes

$$
\Delta n(E)=6.28 \times 10^{-6} P \int_{0}^{\infty} \frac{\Delta \alpha\left(E^{\prime}\right)}{\left(E^{\prime}\right)^{2}-E^{2}} d E^{\prime}-\left(\frac{e^{2} \lambda^{2}}{8 \pi^{2} c^{2} \varepsilon_{0} n}\right)\left(\frac{N}{m_{e}}+\frac{P_{H H}}{m_{h h}}+\frac{P_{L H}}{m_{l h}}\right)
$$

On the whole, the three carrier effects: bandfilling, bandgap shrinkage, and free-carrier absorption, can produce substantial contributions to the total $\Delta \mathrm{n}$. The bandfilling and free-carrier absorption effects both produce a negative $\Delta \mathrm{n}$ for wavelengths in the transparent regime of the semiconductors; bandgap shrinkage produces a positive $\Delta \mathrm{n}$ in the same regime. 


\section{RESULTS AND DISCUSSION}

We have calculated the eigenvalues and eigenfunctions of a SqQW or DFQW. According to Fig. $1 \mathrm{a}$ and $1 \mathrm{~b}$, the results show that as the diffusion length increases, the depth of the well decreases accordingly. However, when the carrier concentrations increase, the well depth increases, which is due to the bandgap shrinkage. By comparing the $\left(\mathrm{E}_{1}-\mathrm{LH}_{1}\right)$ transition energies for different diffusion lengths and carrier concentrations, we can find that the change in diffusion length dominates the change in well depth. Thus the well depth will decrease when the diffusion length and carrier concentration increase simultaneously. In addition, the changes in well width $\mathrm{L}_{z}$ and $\mathrm{Al}$ concentration $\mathrm{x}$ also affects the bandgap shift. We note that if the $\mathrm{Al}$ concentration is increased, the transition energy and well depth will be increased. Moreover, the sensitivity of sp. QW or DFQW to the change in induced carrier concentration will be reduced with the increase in well width. That is to say, the changes in transition energy becomes less sensitive to the changes in diffusion length if the well width is increased. Moreover, when the diffusion length increases, the QW profiles will become effectively more wider, which results in more eigenpairs to be generated.

According to the results shown in Fig.2a and $2 b$, we note that the amplitude of absorption coefficient decreases as the injected carrier concentration increases. The carrier-induced effect, bandfilling, has also been considered when calculating absorption coefficient due to them. This effect causes a reduction in the amplitude of the absorption coefficient with the decrease in the injected carrier concentration. Besides we note that the absorption spectrum for TE mode is significantly different from that for TM mode. For TM mode, both the heavy and light holes are present and the spectrum reproduces all the features observed in the usual geometry. For TM mode, only light holes are observed with increased oscillator strength for which the heavy hole absorption is absent. What is more important, the first upward edge of the absorption coefficients is blue-shifted when the diffusion length increases. However, if the well width is increased, this blue-shift effect induced by different carrier concentrations becomes less significant.

The sign of the change in absorption coefficient $\Delta \alpha$ is always negative, since the absorption coefficient with carrier injection $\Delta \alpha$ is always negative, since the absorption coefficient with carrier injection $\alpha_{N}(N>0)$, is absolutely smaller than that without carrier injection $\alpha_{0}(N=0)$. In addition, since $\alpha_{N}$ decreases with an increase in injected carrier concentrations, we can then understand that $|\Delta \alpha|$ should be larger for cases of higher carrier concentrations. Moreover, according to Fig. 3a and 3b, $\Delta \alpha$ spectrum will be blue-shifted when the diffusion length increases. Just like in the calculation of absorption coefficient, the blue-shift effect induced by different carrier concentrations would be reduced with an increase in well width.

The change in refractive index $\Delta \mathrm{n}$ can be obtained from $\Delta \alpha$ directly by applying the KramersKronig Transformation. According to Fig.4a and $4 \mathrm{~b},|\Delta \mathrm{n}|$ increases when the injected carrier concentration increases. When the photon energy is far less than the bandgap energy, $\Delta \mathrm{n}$ would be very small. As the photon energy approaches the bandgap energy, the amplitude of $\Delta \mathrm{n}$ reaches the peak value. When the photon energy exceeds the bandgap energy, $\Delta \mathrm{n}$ again returns to a small value. In addition, the position of the peak value of $\Delta n$ is dependent on the diffusion length $L_{d}$. It will be blue-shifted if $L_{d}$ 
increases. Moreover, the width has a certain significance on the position of the peak value of $\Delta \mathrm{n}$. If the well width is increased to a larger value, the blue-shift effect induced by different carrier concentrations will become less significant.

\section{ACKNOWLEDGMENTS}

This work is supported in part by the RGC earmarked grant, HKU-CRCG grant, and the City University of Hong Kong Strategic Research Grant.

\section{REFERENCES}

1. E. H. Li and B. L. Weiss, "The optical properties of AlGaAs/GaAs hyperbolic quantum-well structures," J. Appl. Phys., vol. 70, pp. 1054-1056, 1991.

2. E. H. Li and B. L. Weiss, "Dielectric properties of nonsquare AlGaAs/GaAs single quantum wells at photon energies below the bandgap," J. Appl. Phys., vol. 59, pp. 3312-3314, 1991.

3. B. R. Bennett, R. A. Soref, J. A. Del Alamo, "Carrier-Induced Change in Refractive Index of InP, GaAs, and InGaAsP," IEEE J. Quantum Electron., vol. 26, pp. 113-122, 1990.

4. A. Tourita and A. Suzuki, "Carrier-Induced Lasing Wavelength Shift for Quantum Well Laser Diodes," IEEE J. Quantum Electron., vol. QE-23, pp. 1155-1159, 1987.

5. E. H. Li and B. L. Weiss, "Exciton Optical Absorption in a Diffusion Induced Non-Square AlGaAs/GaAs Quantum Well," Quantum Well and Superlattice Physics IV, vol. 1675, pp. 98-108, 1992.

6. S. L. Chuang and L. Ahn, "Optical transitions in a parabolic quantum well with an applied electric field analytical solutions," J. Appl. Phys., vol. 65, pp. 2822-2826, 1989.

7. F. Stern and S. Das Sarma, "Electron energy levels in GaAs-Ga ${ }_{1-X} \mathrm{Al}_{\mathrm{X}} \mathrm{As}$ heterojunctions," Phys. Rev., vol. B30, pp. 840-848, 1984.

8. J. Micallef, E. H. Li, and Bernard L. Weiss, "Exciton Optical Absorption in Disordered, Strained InGaAs/GaAs Single Quantum Wells," Superlattices and Microstructures, vol. 13, pp. 315-322, 1993.

9. H. C. Huang, S. Yee, and M. Soma, "Quantum Calculations of the change of refractive index due to free carriers in silicon with nonparabolic band structure," Appl. Phys., vol. 67, pp. 2033-2039, 1990 

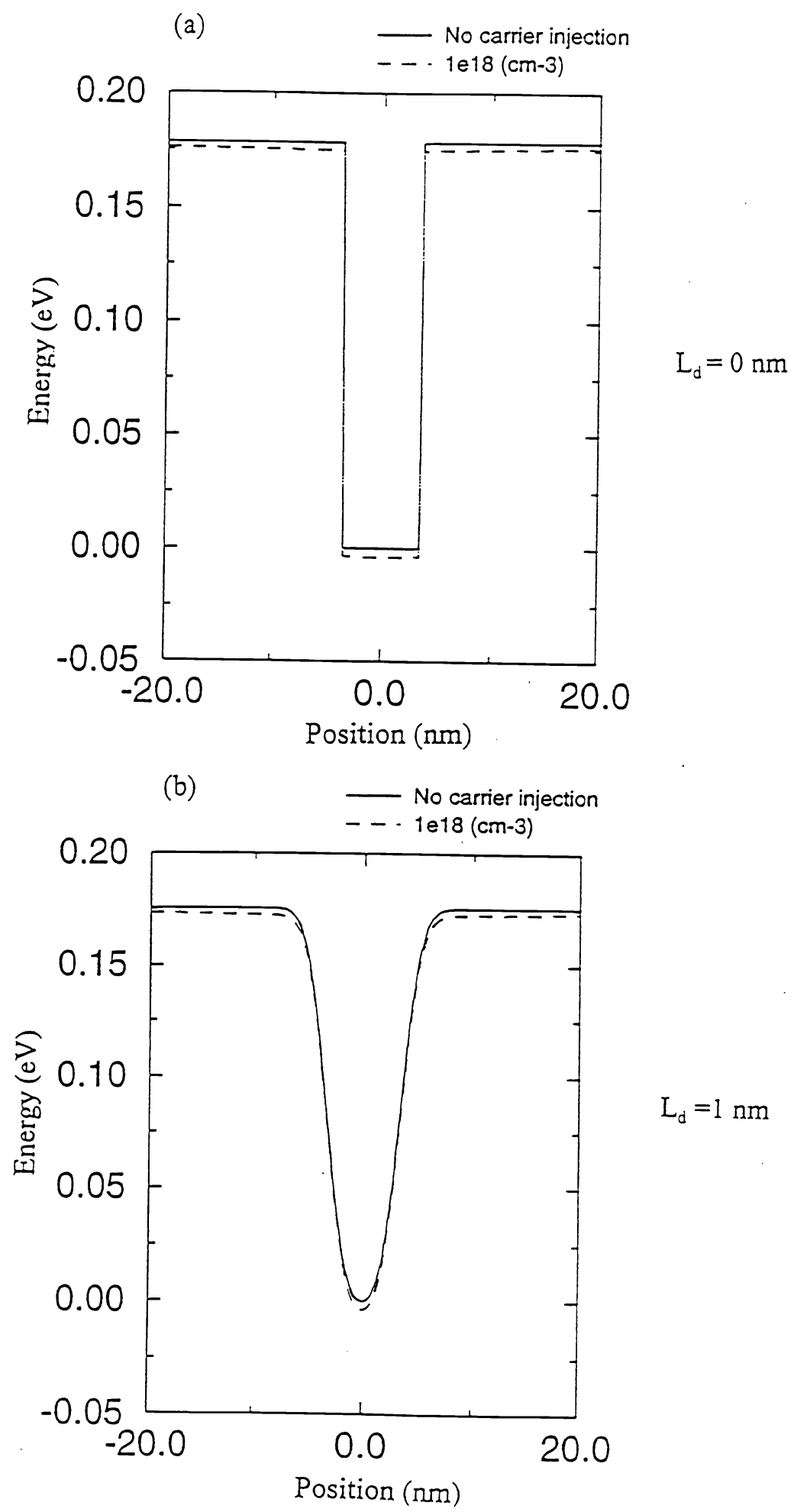
Fig. 1 shows the confinement profile for electrons when the well width $L_{2}=7 \mathrm{~nm}$. Al concentration
$=0.2$ 
(a)

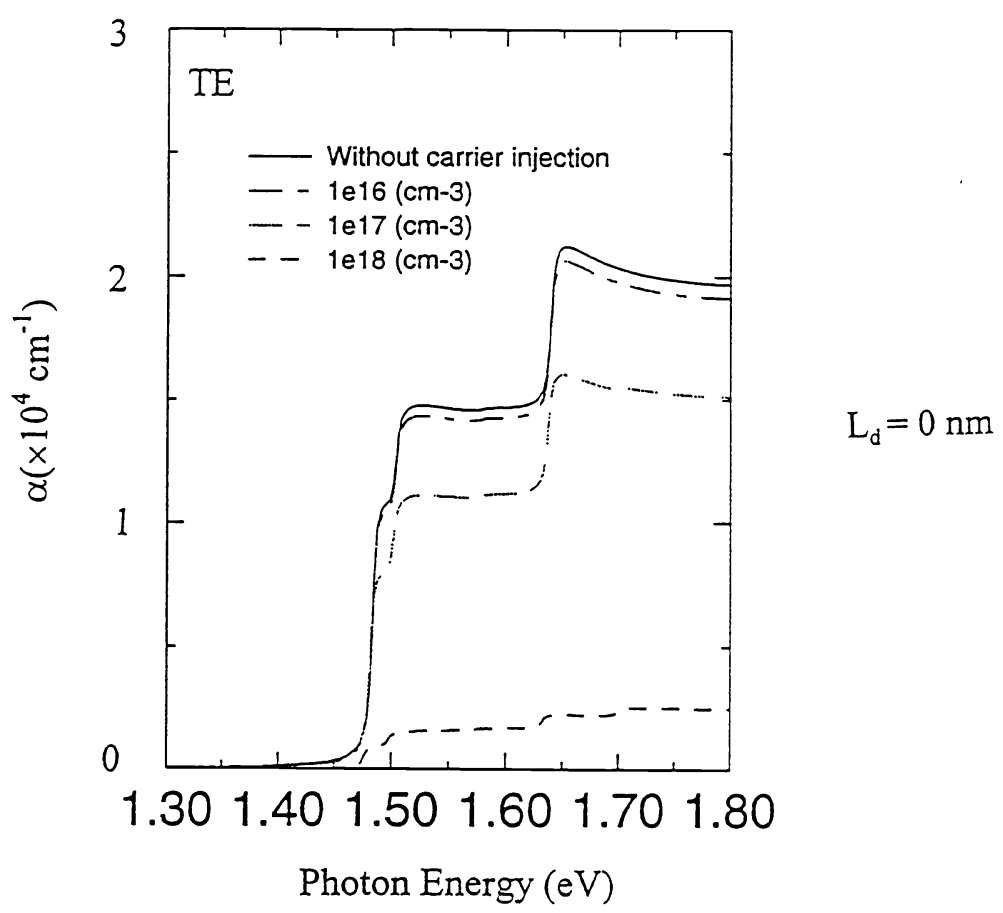

(b)

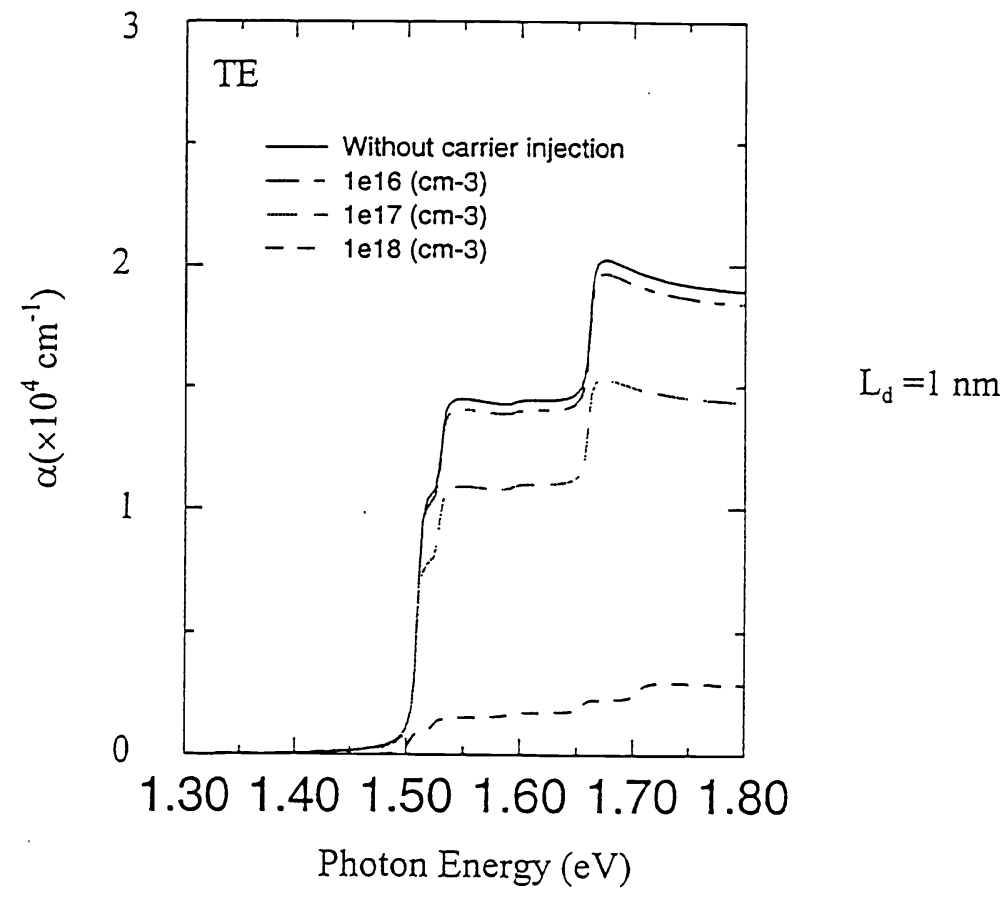

Fig. 2 shows the absorption coefficient $\alpha$ spectrum (TE mode) when the well width $\mathrm{L}_{\mathrm{Z}}=7 \mathrm{~nm}, \mathrm{Al}$ concentration $=0.2$ 
(a)

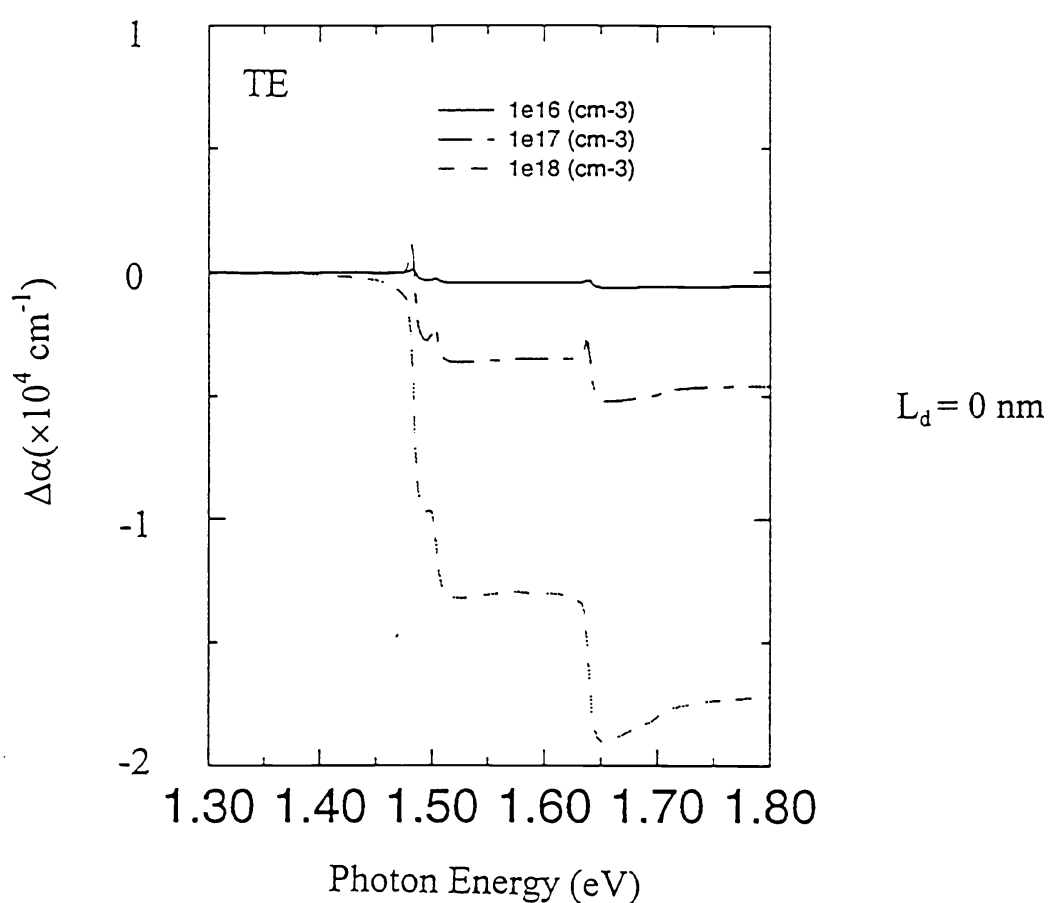

(b)

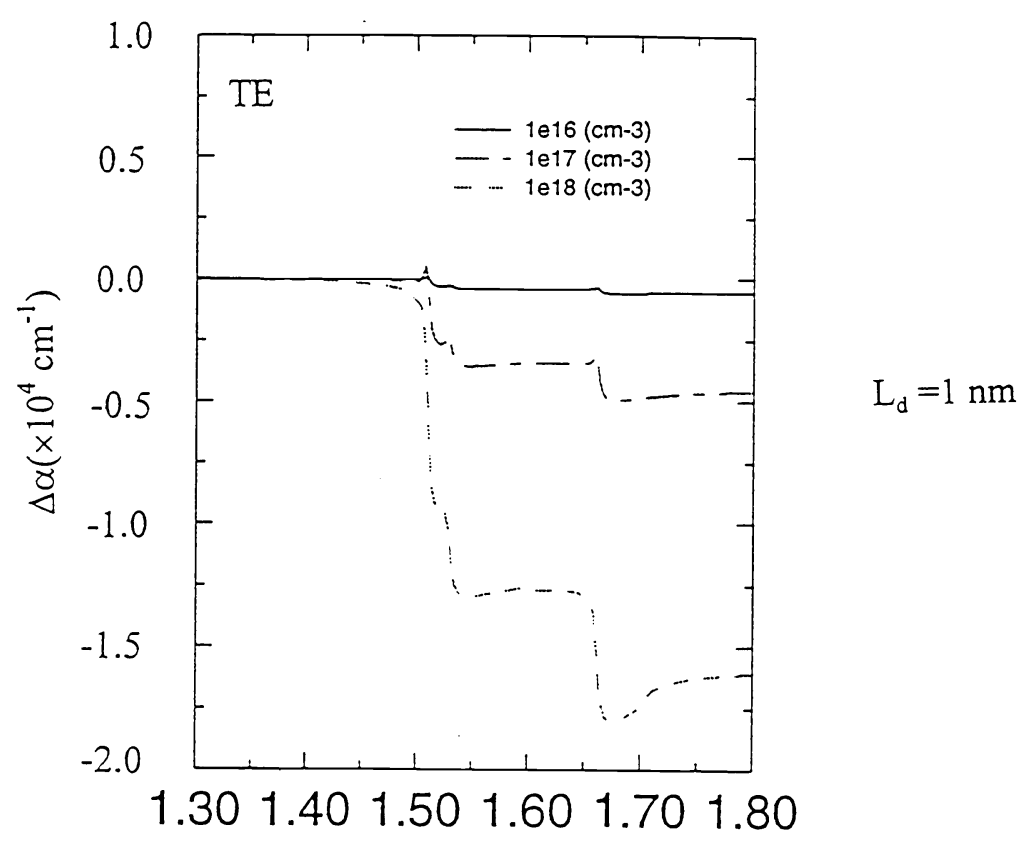

Photon Energy (eV)

Fig. 3 shows the change in absorption coefficient $\Delta \alpha$ spectrum (TE mode) when the well width $\underline{\mathrm{L}}_{2}=7 \mathrm{~nm}, \mathrm{Al}$ concentration $=0.2$ 
(a)

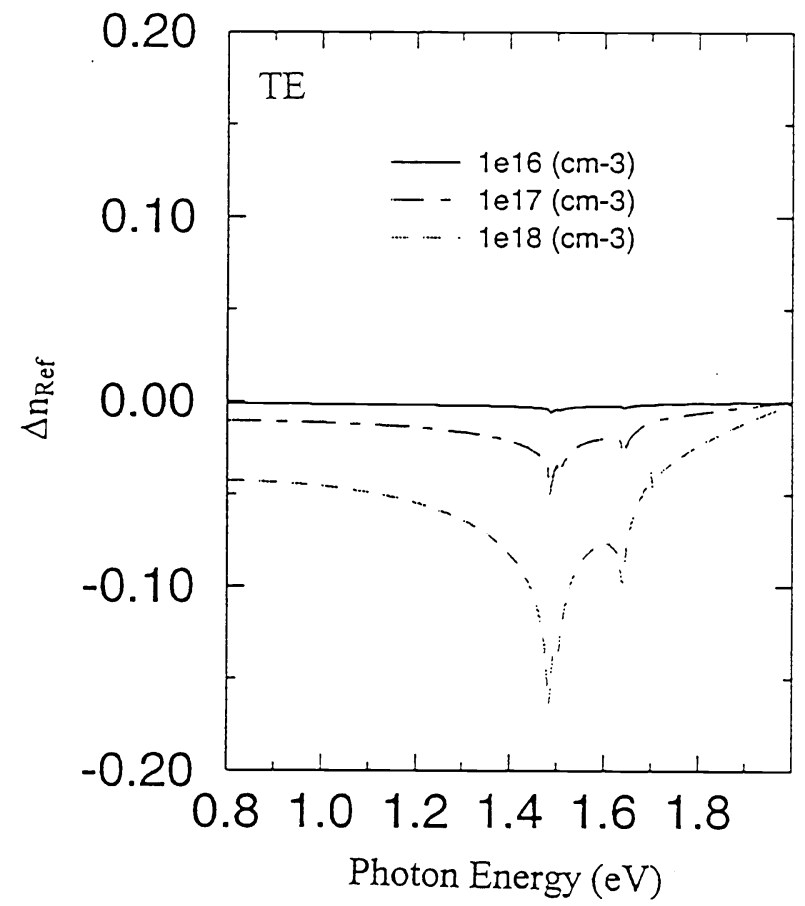

(b)

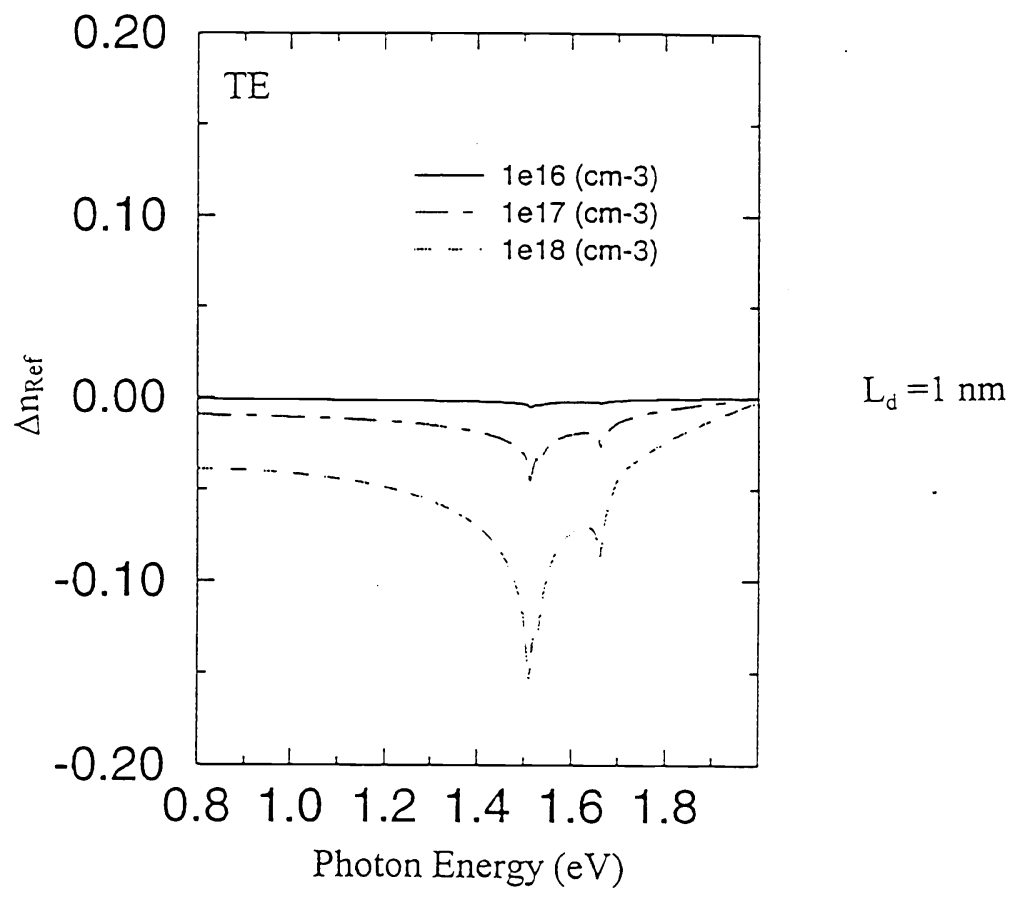

Fig. 4 shows the change in refractive index $\Delta \mathrm{n}$ spectrum (TE mode) when the well width $\mathrm{L}_{Z}=7 \mathrm{~nm}$. $\underline{\text { Al concentration }=0.2}$ 